\title{
¿ES EL ESPAÑOL UNA LENGUA S-V-O?
}

1. En particular desde Greenberg se viene clasificando el español, en la tipología que se sitúa al nivel de los elementos constitutivos de la oración, entre el grupo en que domina la secuencia lineal SVO, es decir "objeto-verbo-objeto", como en:

\section{Mi hermana / compró / un coche \\ $\mathrm{S}$ \\ $\mathrm{V}$ \\ $\mathrm{O}$}

Hay que añadir en seguida que otras combinaciones son usuales, como VSO, y según las circunstancias aparecen incluso las seis combinaciones posibles. Naturalmente esto vale cuando se toman como base las oraciones transitivas en tercera persona con el sujeto explícito. Pero sabemos que:

(a) El español no necesita del sintagma nominal sujeto cuando ya se conoce:

$$
\begin{gathered}
\text { Compró / un coche } \\
\mathrm{V}
\end{gathered}
$$

¿Vamos a decir que la oración es del tipo VO?

(b) La situación fundamental de la comunicación es la que se desarrolla entre dos personas y en ese caso no se suelen expresar los pronombres de primera y segunda persona:

$$
\begin{gathered}
\text { Compré / un coche } \\
\text { ¿Vas a comprar / un coche? }
\end{gathered}
$$

De modo que si tomamos en cuenta los contextos oracionales y la frecuencia de las situaciones de diálogo, podemos afirmar que 
la secuencia VO es la más usual en español, por lo menos aparentemente. En las mismas condiciones el francés presenta el orden SVO por la obligación de la expresión del pronombre personal sujeto.

2. Si examinamos ahora la composición del núcleo de la oración transitiva básica de primera y segunda persona:

$$
\begin{gathered}
\text { (Ayer) compré un coche } \\
\text { Lo compré (ayer) }
\end{gathered}
$$

vemos que el español usa tres clases de morfemas:

(i) Un morfema lexical (o lexema) que puede entrar en la formación

- de un verbo, y viene siempre acompañado de un morfema gramatical, que en nuestro ejemplo incluye la categoría nominal de sujeto: compr-(e)

- de un sustantivo (o de un adjetivo), el cual puede ser sustituido por un pronombre: coche $>$ lo.

(ii) Un morfema gramatical ligado, el $-\hat{e}$ ya mencionado.

(iii) Un morfema gramatical libre, el pronombre $l o$.

De ahí que la forma conjugada del verbo en español tenga que transcribirse con Vs (lexema verbal con sujeto incorporado) y el objeto pronominal con $\mathrm{O}$ - (-O si va pospuesto).

$\mathrm{Si}$ volvemos a los ejemplos ya citados, escribiremos:

$\begin{array}{rrrr}\text { Mi hermana compró un coche } & \mathrm{S} & \mathrm{Vs} & \mathrm{O} \\ \text { compró un coche } & \mathrm{Vs} & \mathrm{O} \\ \text { compré un coche } & \mathrm{Vs} & \mathrm{O} \\ \text { lo compré } & \mathrm{O}-\mathrm{Vs} & \end{array}$

Se ve que se está muy lejos de una lengua SVO, como lo es el francés con ma soeur a acheté une voiture, elle a acheté une voiture, j'ai acheté une voiture, je l'ai achetée (se trata siempre de $\mathrm{S}-\mathrm{Vs}-\mathrm{O}$ ).

3. Habría que considerar también la oración con tres actantes, que incluye al destinatario (dativo, beneficiario), simbolizado por D:

$$
\begin{array}{cccc}
\text { Juan entregó el libro a } & \text { María } \\
\mathrm{S} & \mathrm{Vs} & \mathrm{O} & \mathrm{D}
\end{array}
$$


realizado corrientemente como

$$
\begin{array}{ccc}
\text { entregó el libro a } & \text { María } \\
\text { Vs } & \text { O } & \text { D }
\end{array}
$$

y con las anáforas usuales:

$$
\begin{array}{llll}
\text { lo entregó a Maria } & \mathrm{O}- & \mathrm{Vs} & \mathrm{D} \\
\text { le entregó el libro } & \mathrm{D}- & \mathrm{Vs} & \mathrm{O} \\
\text { se lo entregó } & \mathrm{D}- & \mathrm{O}- & \mathrm{Vs}
\end{array}
$$

También puede haber doble expresión de un actante:

$$
\begin{array}{ll}
\text { te daré a ti más dinero } & \mathrm{D}^{1}-\mathrm{Vs} \mathrm{D}^{2} \mathrm{O} \\
\text { no le diré nada a Juan } & \mathrm{D}^{1}-\mathrm{V} \mathrm{O} \mathrm{D}^{2}
\end{array}
$$

Según la intención en el mensaje (cfi la entonación), el segundo elemento puede ser una topicalización $(\mathrm{T})$ :

$$
\text { no le diré nada, a Juan } \quad \text { D- Vs O } \mathrm{T}_{\mathrm{d}}
$$

En el caso de la primera y la segunda personas:

$$
\text { yo compré un coche, tú no lo compraste }
$$

se trata en español de una topicalización, de modo que tampoco tenemos el orden $\mathrm{SVO}$, sino $\mathrm{T}_{\mathrm{S}} \mathrm{Vs} \mathrm{O}$ y $\mathrm{T}_{\mathrm{S}} \mathrm{O}-\mathrm{Vs}$.

En "lo compré yo" sería: O- Vs $\mathrm{T}_{\mathrm{S}}$.

Ejemplos de secuencias más complejas:

$$
\begin{gathered}
\text { Mira, yo, este coche, a Juan, se lo vendi hace un mes } \\
\mathrm{T}_{\mathrm{S}} \mathrm{T}_{\mathrm{o}} \mathrm{T}_{\mathrm{d}} \mathrm{D}-\mathrm{O}-\mathrm{V} \mathrm{s}
\end{gathered}
$$

4. Con los verbos intransitivos, tampoco es el orden SV el más usual en español:

$\begin{array}{lrl}\text { 'gimió el muchacho } & \mathrm{Vs} & \mathrm{S} \\ \text { se durmió } & \mathrm{s} \mathrm{s} & \text { (pronominal) } \\ \text { acepté } & \mathrm{VS} & \\ \text { entraron los alumnos } & \mathrm{VS} & \mathrm{S} \\ \text { ¿encontraste? } & \mathrm{Vs} & \end{array}$


En las formas verbales que exigen la posposición, se encuentran los mismos fenómenos:

$$
\begin{array}{ll}
\text { dámelo a mí } & \mathrm{VS}-\mathrm{D}^{1}-\mathrm{O}^{2} \\
\text { sabiéndolo yo } & \mathrm{V}-\mathrm{O}
\end{array}
$$

Con ciertos auxiliares (W), hay dos soluciones:

$$
\begin{array}{ll}
l o \text { puedo hacer } & \mathrm{O}-\mathrm{Ws} \mathrm{V} \\
\text { puedo hacerlo } & \mathrm{W} \mathrm{V}-\mathrm{O}
\end{array}
$$

Otros muchos comportamientos aparecerían, al considerar la lengua popular y el fenómeno de la subordinación, o el de la interrogación.

5. En resumen, se puede afirmar que el español no es una lengua SVO. El "tipo" sería Vs O del que se deduciría:

$\begin{array}{clll}\operatorname{actantes} & 1^{\mathrm{a}} & 2^{\mathrm{a}} & 3^{\mathrm{a}} \\ 2 & \mathrm{Vs} & \mathrm{O} & (\mathrm{S}) \mathrm{Vs} \mathrm{O} \\ 1 & \mathrm{Vs} & & (\mathrm{S}) \mathrm{Vs}(\mathrm{S}) \\ 3 & \mathrm{Vs} & \text { O D } & (\mathrm{S}) \mathrm{Vs} \mathrm{O} D\end{array}$

Con pronominalización:

$$
\left.\left.\begin{array}{l}
\text { O- } \\
\text { D- } \\
\text { D- O- }
\end{array}\right\} \text { Vs } \quad \text { (y posibilidad de }-\mathrm{O} \text { y }-\mathrm{D}\right)
$$

Con topicalización:

$\mathrm{T}_{\mathrm{s}}, \mathrm{T}_{\mathrm{o}}, \mathrm{T}_{\mathrm{d}}$ con orden subjetivo.

Con focalización: "YO se lo di" (y no otra persona): $F_{S}$

Ejemplo con complejidad y ambigüedad:

$$
\begin{array}{lllll}
Y_{0} & \text { se } & \text { lo daréi } & \text { el libro } & \text { a María } \\
\mathrm{T}_{\mathrm{S}} & \mathrm{D}^{1}-\mathrm{O}^{1}-\mathrm{Vs} & \mathrm{O}^{2} & \mathrm{D}^{2} \\
\mathrm{~F}_{\mathrm{S}} & \cdot- & \mathrm{T}_{\mathrm{O}} & \mathrm{T}_{\mathrm{d}}
\end{array}
$$


El comportamiento pronominal tiene tanto valor, desde el punto de vista tipológico, como el de los sintagmas explícitos. En muchas lenguas, la forma verbal implica la presencia de ciertas clases de pronombres; en este caso las fórmulas son del tipo "oVs" o "sVo", etc. Incluso en nuestras lenguas indoeuropeas hay que revisar la presentación de los elementos constituyentes de la oración.

Bernard Pottier Universidad de París, Sorbona 